\title{
Middle Cranial Fossa Approach: The Incudomalleolar Joint as a Reliable Landmark
}

\author{
Syed Salman Hashmi ${ }^{10}$ Juan Carlos Izquierdo ${ }^{2(0)}$ Susan D. Emmett ${ }^{3,4}$ Thomas Edwin Linder ${ }^{5(0)}$ \\ ${ }^{1}$ Department of Otolaryngology, Baqai Medical University, Karachi, \\ Sindh, Pakistan \\ 2 Department of Surgery, Division of Otolaryngology, Universidad \\ Nacional de Colombia, Bogota, Colombia \\ ${ }^{3}$ Department of Head and Neck Surgery and Communication Sciences, \\ School of Medicine, Duke University, Durham, NC, United States \\ ${ }^{4}$ Duke Global Health Institute, Duke University, Durham, NC, United States \\ ${ }^{5}$ Department of Otorhinolaryngology, Luzerner Kantonsspital, \\ Luzern, Switzerland \\ Address for correspondence Thomas Edwin Linder, MD, Department \\ of Otorhinolaryngology, Luzerner Kantonsspital, Spitalstrasse, \\ Luzern 6000, Switzerland (e-mail: Thomas.Linder@luks.ch). \\ Int Arch Otorhinolaryngol 2021;25(3):e392-e398.
}

\begin{abstract}
Keywords

- middle cranial fossa surgery

- transtemporalsupralabyrinthine approach

- superior semicircular dehiscence

- arcuate eminence

Introduction The middle cranial fossa approach is performed by fewer neurotologists owing to a reduced number of indications. Consistent landmarks are mandatory to guide the surgeon in a narrow field.

Objectives We have evaluated the incus and malleus head and the incudomalleal joint (IMJ) as a key landmark for identifying the superior semicircular canal (SSC) and to get oriented along the floor of the middle cranial fossa.

Methods A combination of 20 temporal bone dissections and CT imaging were utilized to test and describe these landmarks.

Results The blue line of the SSC is consistently identified along the prolongation of a virtual line through the IM] and the angulation toward the root of zygoma. The mean distance from the zygoma toward the IMJ ranged from 1.60 to $1.90 \mathrm{~cm}$. Once the IM] was identified, the blue line of the SSC was consistently found along the virtual line through the IMJ within 5 to $9 \mathrm{~mm}$.

Conclusions The IMJ is a safe and consistent anatomical marker in the surgical approach to the middle cranial fossa floor. Opening the tegmen 1.5 to $2 \mathrm{~cm}$ medial to the root of the zygoma and identifying the joint allows to trace a virtual line toward the SSC within 5 to $9 \mathrm{~mm}$. Knowledge of the close relationship between the direction of the IMJ and the superior canal can be used in all transtemporal approaches, thus orienting the surgeon in a rather narrow field with limited retraction of the dura and brain.
\end{abstract}

\section{Introduction}

The middle cranial fossa approach (MCFA) is currently performed by fewer neurotologists owing to a reduced number of indications. However, it is still widely accepted as a standard surgical approach for several pathologies,

received

November 3, 2019

accepted

March 19, 2020

published online

September 24, 2020
DOI https://doi.org/ 10.1055/s-0040-1712105. ISSN 1809-9777. including facial nerve decompression for Bell palsy or temporal bone fractures, ${ }^{1}$ petrous apex cholesteatomas, ${ }^{2}$ and acoustic neuromas with limited cerebellopontine angle (CPA) involvement. ${ }^{3}$ A more recent addition to the indications is the repair of superior semicircular canal dehiscence (SSCD). ${ }^{4}$

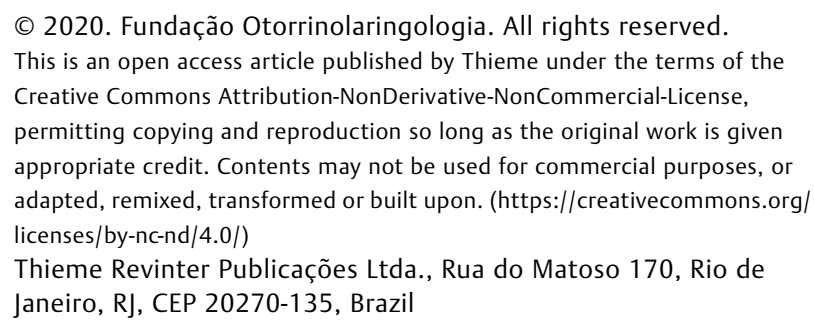


Identification of the superior semicircular canal (SSC) has been a key feature for most middle cranial fossa surgeries due to the consistent relationship to the internal auditory canal. ${ }^{5,6}$ Conventionally, the arcuate eminence is considered to be the protrusion of SSC into the middle fossa; however, numerous studies have negated this concept. ${ }^{7,8}$ In SSCD, where correct identification of the superior canal is the most important landmark, multiple honeycomb-like tegmen defects ${ }^{9}$ are frequently reported ${ }^{10}$ that may mimic a canal dehiscence and, therefore, lead to a misidentification of the SSC.

Due to these challenges, junior skull base surgeons may find difficulties in identifying the SSC in the middle cranial fossa floor. In the present study, we have evaluated the incus and malleus head, as well as the incudomalleal joint (IMJ), as an important landmark for identifying the SSC. A combination of temporal bone dissection and computed tomography (CT) imaging were utilized to test and describe these landmarks.

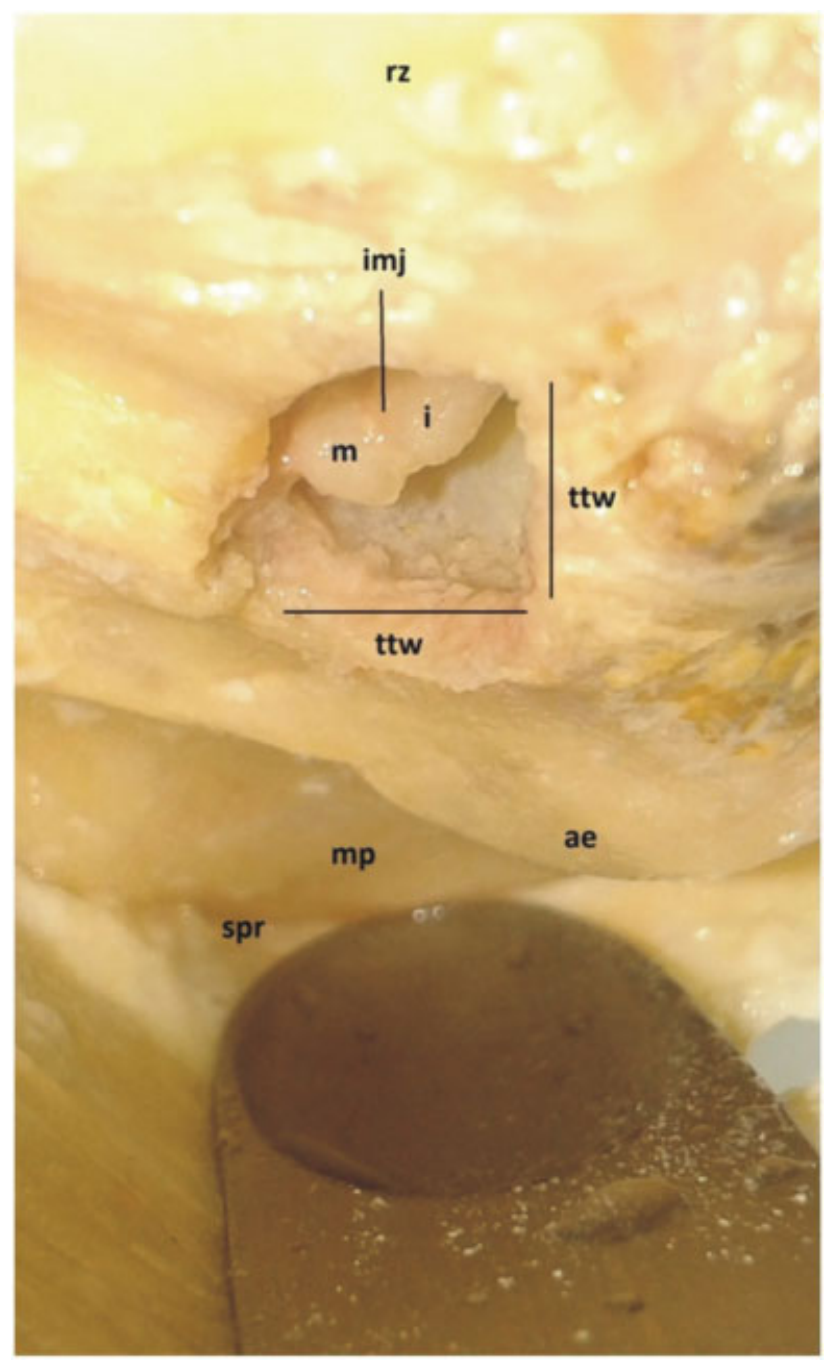

Fig. 1 Tegmen tympani window. Abbreviations: ae, arcuate eminence; i, Incus; imj, incudomalleal joint;m, malleus; mp; meatal plane; rz, root of zygoma; spr, superior petrosal ridge;ttw, tegmen tympani window.

\section{Materials and Methods}

Twenty temporal bones were dissected and documented photographically. The bones were dissected in the usual surgical position. First, a craniotomy was performed on the temporal bone squama centered at the root of the zygoma (ROZ) and the external ear canal (EAC), as described by Fisch. ${ }^{6}$ The floor of the MCF was exposed with limited retraction of the dura. The arcuate eminence was then identified, and a self-retaining retractor was placed just medial to it. The head of the malleus and a portion of the body of the incus were exposed by drilling a window at the tegmen tympani, in between the root of the zygoma laterally and the arcuate eminence medially ( - Fig. 1) Once the IMJ was identified, the dissector then visualized the root of zygoma (ROZ). A line is projected through the IMJ posteriorly toward the superior petrosal ridge (-Fig. 2). The SSC is dissected along the projected line to further identify the yellowish color of the SSC and then the blue line of the SSC itself ( - Fig. 3). Finally, as described by Fisch, ${ }^{6}$ a line is also drawn 60 degrees anteriorly from the ampullary end of the SSC to further identify the internal auditory canal (IAC) from the porus to the fundus, exposing the meatal foramen, the labyrinthine portion of the facial nerve, the geniculum, the proximal tympanic facial nerve ( $\boldsymbol{- \text { Fig. }} \mathbf{4}$ ), and the internal

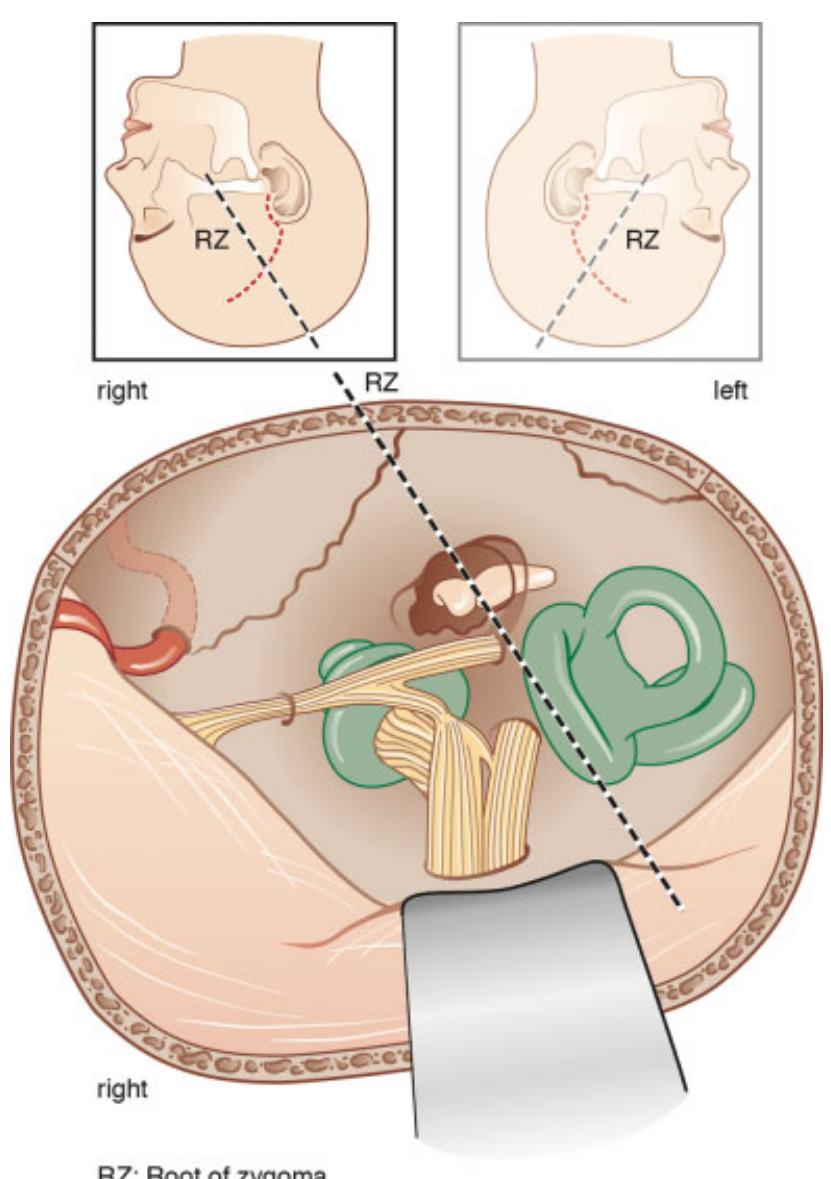

Fig. 2 Schematic representation of consistent landmarks at the floor of the middle cranial fossa. 


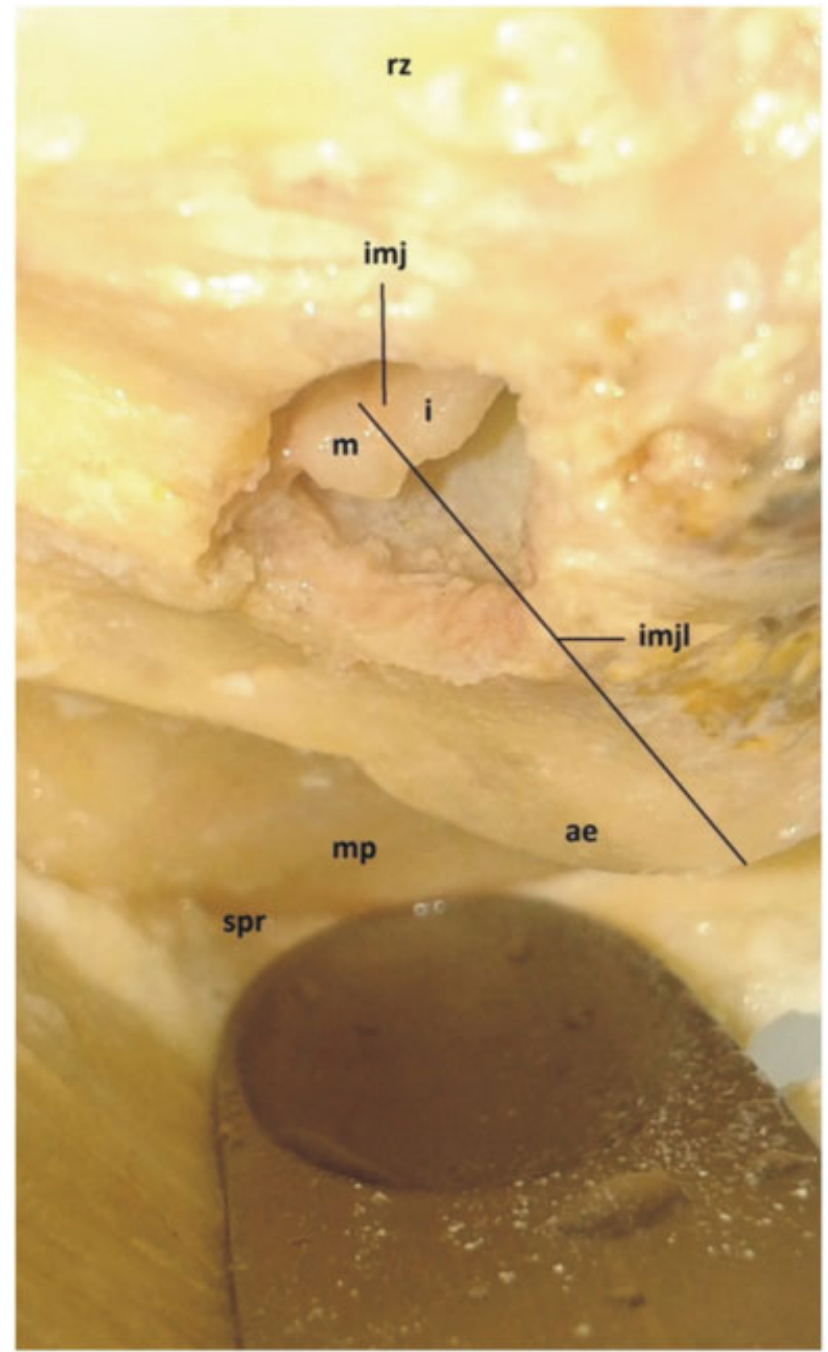

Fig. 3 Projection of the incudo-malleal joint line toward the arcuate eminence. Abbreviations: ae, arcuate eminence; i, incus; imj, incudomalleal joint; imjl:,incudomaleal joint line; $m$, malleus;mp,meatal plane; rz, root of zygoma; sps, superior petrosal ridge.

auditory canal ( - Fig. 5). The distance from the IMJ to the root of the zygoma was measured with a millimetric caliper.

Upon ethical approval, the radiological portion of the study consisted of measurements on anonymized CT scans in 20 temporal bones of 10 patients with suspected otosclerosis. The Picture Archiving and Communication System (PACS) version 5.5.168468 from Phönix-PACS GmbH (Freiburg, Germany) was used for image visualization, reconstruction, and measurements. Measurements were taken from the midpoint of the IMJ to the superior most point of the SCC along the MCF, using axial (-Fig. 6) and paracoronal images (-Fig. 7).

\section{Results}

The SSC and its blue line were consistently identified along the prolongation of a virtual line through the incudomalleal joint and the angulation toward the root of zygoma of the patient. Means of measurements are shown in - Table 1. The mean distance taken from to the ROZ toward the IMJ ranged from 1.60 to $1.90 \mathrm{~cm}$. The mean window area of the tegmen

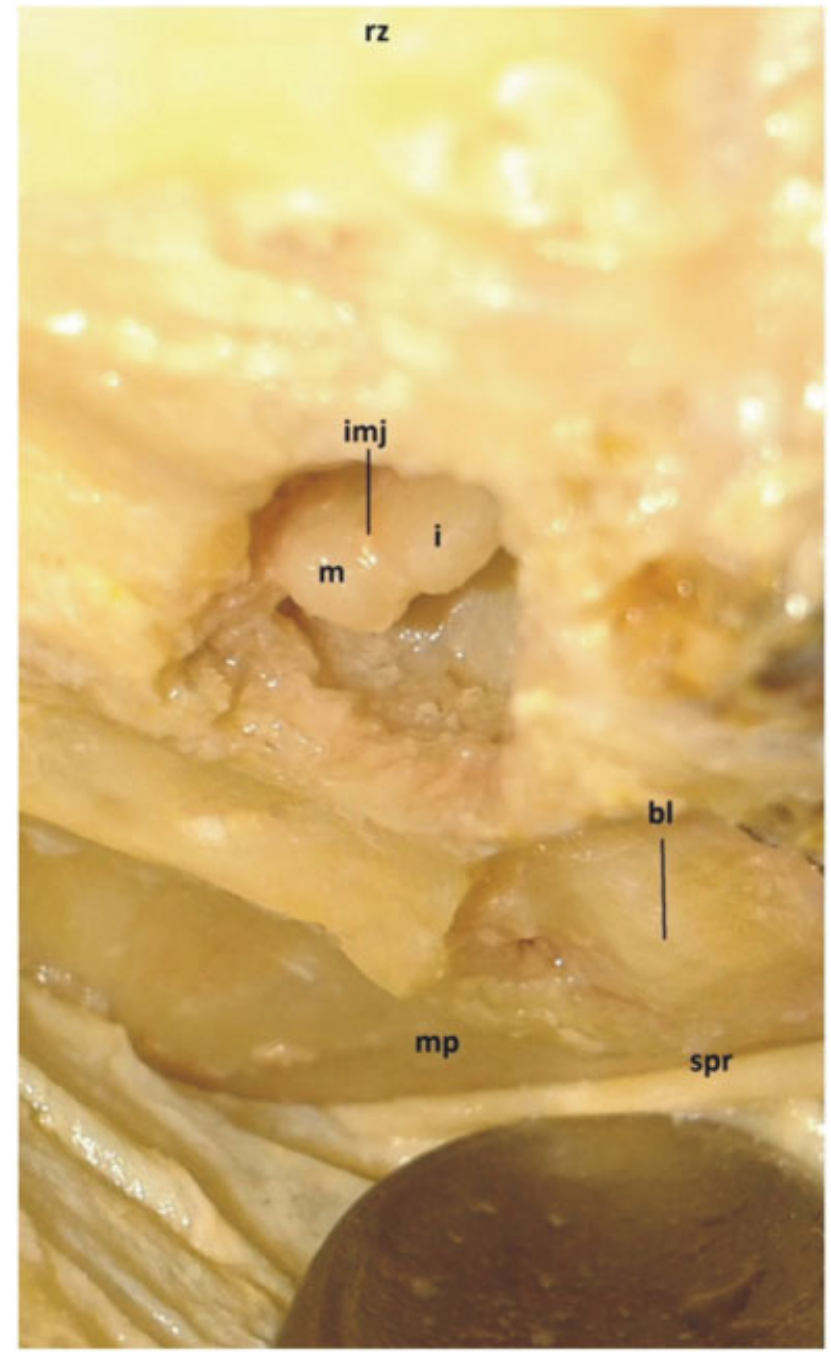

Fig. 4 Blue line of the superior semicircular canal. Abbreviations: bl, blue line of the superior semicircular canal i, incus; imj, incudomalleal joint;m, malleus; mp, meatal plane; rz, root of zygoma; spr, superior petrosal ridge.

tympani was $59 \mathrm{~mm}^{2}$ (SD 29.07), with a 7 to $8 \mathrm{~mm}$ diameter of the oval shaped opening. The blue line of the SCC was consistently found along a virtual line from the ROZ through the IMJ in all the 20 temporal bones as shown in the diagram.

Radiological distance measurements between the midpoint of the IMJ and the superior most part of the SSC in axial scans ranged from 6.4 to $8.9 \mathrm{~mm}$ (mean: $7.74 \mathrm{~mm}$, standard deviation [SD]: 0.66). In reconstructed paracoronal images, the distances varied from 5.17 to $7.6 \mathrm{~mm}$ (mean: 6.87, SD: 0.72).

\section{Discussion}

The orientation along the MCF floor is challenging due to multiple factors, such as the small target area, close proximity of vital structures (e.g., facial nerve, cochlea and labyrinth), increased bleeding in the event of extended exposure, and the depth of the surgical field. The probable presence of multiple dehiscences in critical areas, like the geniculate ganglion and the internal carotid artery, can further contribute to the technical difficulties of middle fossa surgery. ${ }^{10,11}$ 


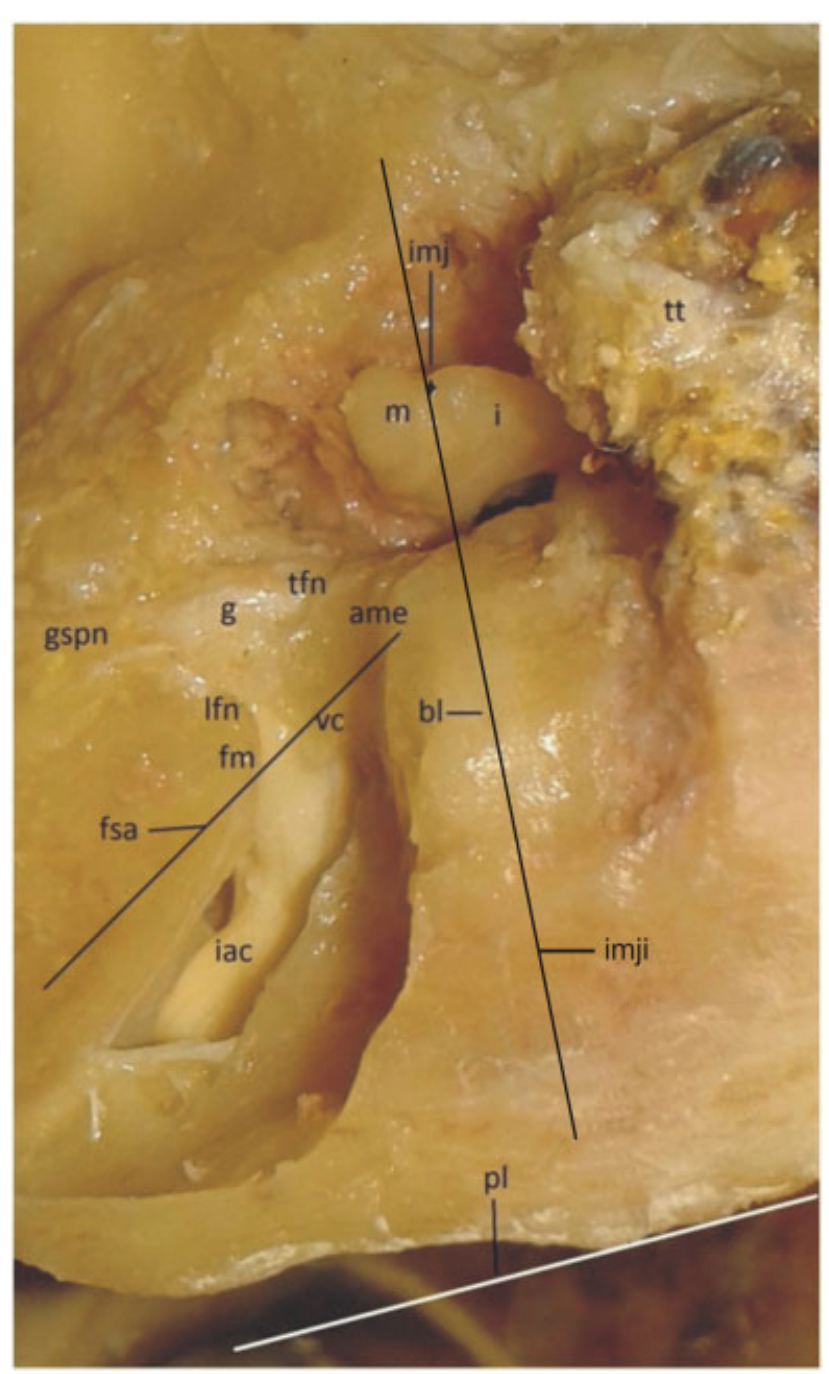

Fig. 5 Identification of the Internal auditory canal and facial nerve. Abbreviations: ame, ampullary end of superior semicircular canal; bl, blue line of the superior semicircular canal; fm, foramen meatale; fsa, 60 degree angle line from the ampullary end of the superior semicircular canal; g, geniculate ganglion; gspn, greater superficial petrosal nerve; i, incus; iac, internal auditory canal; imj, incudomalleal joint; imjl, incudomalleal joint line crossing the blue line of superior semicircular canal; Ifn, labyrinthine facial nerve segment; $\mathrm{m}$, malleus; $\mathrm{pl}$, petrosal line; $\mathrm{tfn}$, tympanic facial nerve segment; $\mathrm{tt}$ : tegmen tympani; vc, vertical crest.

Many surgeons take the arcuate eminence as an initial landmark in the MCFA before proceeding to other structures. However, the presence of multiple humps of bone along the middle fossa floor, and an inconsistent relationship of the arcuate eminence with the $\mathrm{SCC}^{3,4}$ make this landmark highly unreliable. These technical challenges have also led surgeons to rely on expensive navigation systems for exact orientation in the MCF. Notably, these systems are not present in many centers worldwide. Personal experience of the senior author with optical tracing systems has also revealed difficulties with properly mounting reference points so that they are always visible to the cameras, and electromagnetic systems may not provide sufficient resolution at the depth of surgical dissection.
These challenges highlight the need for a reliable and safe landmark in the middle fossa floor that can orient the surgeon toward critical structures. In the present study, we have identified the IMJ as a very consistent landmark to orient the surgeon toward the exact location of the SSC. The most direct approach to identifying the ossicles at the floor of the MCF is the combined transmastoid-transtemporal approach. Mastoidectomy provides access to perform a burr hole just above the incus body and malleus head via the lateral route. By changing the position of the microscope subsequent to the craniotomy, the IMJ can be easily identified at the MCF floor. The indications for using a combined approach include facial nerve decompression for longitudinal fractures of the temporal bone or repair of tegmen defects. In the pure middle fossa approach for other indications, we suggest that after craniotomy and placement of the retractor medial to the arcuate eminence, the surgeon should first identify the IMJ by opening the tegmen $\sim 1.5$ to $2.0 \mathrm{~cm}$ from the ROZ and lateral to the arcuate eminence. The analysis of the coronal CT scans helps in the proper orientation. In case of doubt, the use of an angled endoscope looking through a small hole in the tegmen may help to identify the precise location of incus and malleus before further drilling. This additional step may also prevent the surgeon from touching the ossicles with the drill and inducing inner ear trauma.

Once the incus body and malleus head are identified, the blue line or dehiscence of the SSC can then be traced in a line from the ROZ to the IMJ posteriorly. Longitudinal (along this imaginary line) and rotatory drill movements using diamond drills allow to shape out the SSC within the arcuate eminence. Our radiological measurements show that the highest point of the SCC is less than $1 \mathrm{~cm}$ from the IMJ (range: $5.52-8.85 \mathrm{~mm}$ in our study). If the surgery requires identification of the IAC, this structure can be traced along the 60degree angle anterior to the blue line of the SSC, as described by Fisch. ${ }^{5,6}$ Subsequently, the tegmen defect is closed with a piece of calvarial bone. The bone should be placed in a way that the concave side should be facing the ossicles, making sure it is not touching the ossicles.

Other studies have also provided radiologically evident landmarks to locate the SCC dehiscence. Patel et al reported the SSC to be within 1 inch $(2.54 \mathrm{~cm})$ from the edge of the middle fossa craniotomy centered over the EAC. ${ }^{10} \mathrm{~A}$ similar study was also published by Joel el al. ${ }^{12}$ They took the midpoint of the EAC at the level of the zygoma as a reference point to locate the SCC. However, both of these studies were radiological and have not showed enough surgical or temporal bone dissection data to support their practical application. Second, we urge identification of the entire blue line of the SCC instead of relying on a single point as a landmark. The line from the ROZ through the IMJ facilitates identification of the entire blue line of the SCC, making identification of this important landmark safe and reliable. Careful evaluation of the preoperative coronal CT scan is, nevertheless, essential for surgical planning. The degree of pneumatization above the superior canal allows the surgeon to visualize the height and width of the arcuate eminence and allows estimating the amount of 

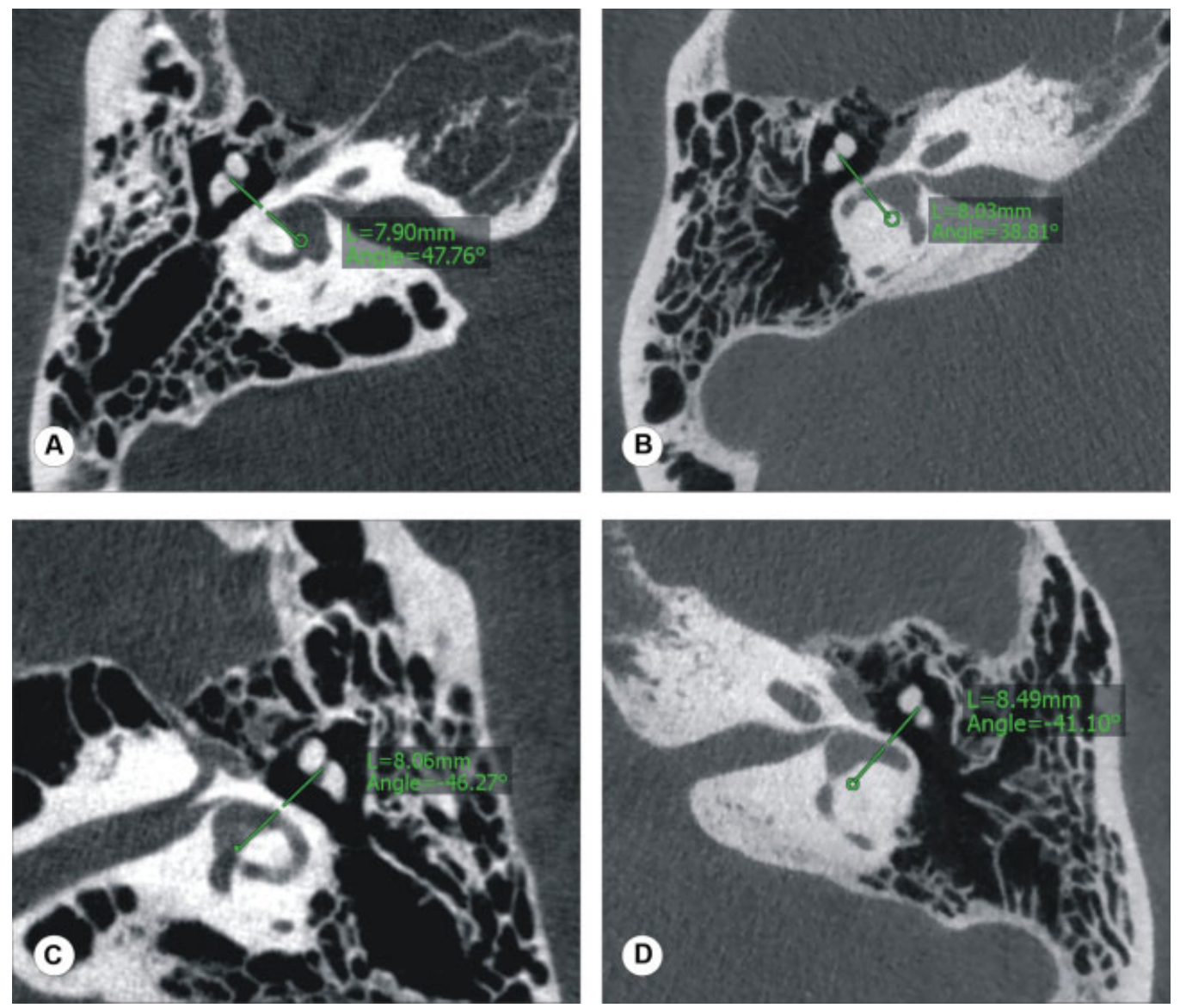

Fig. 6 Measurement of distance between the SCC and the IMJ in axial scans. Abbreviation: L: distance between the SCC midpoint (marked first with circle) and the midpoint of the IMJ (by scrolling the scans to the joint level).
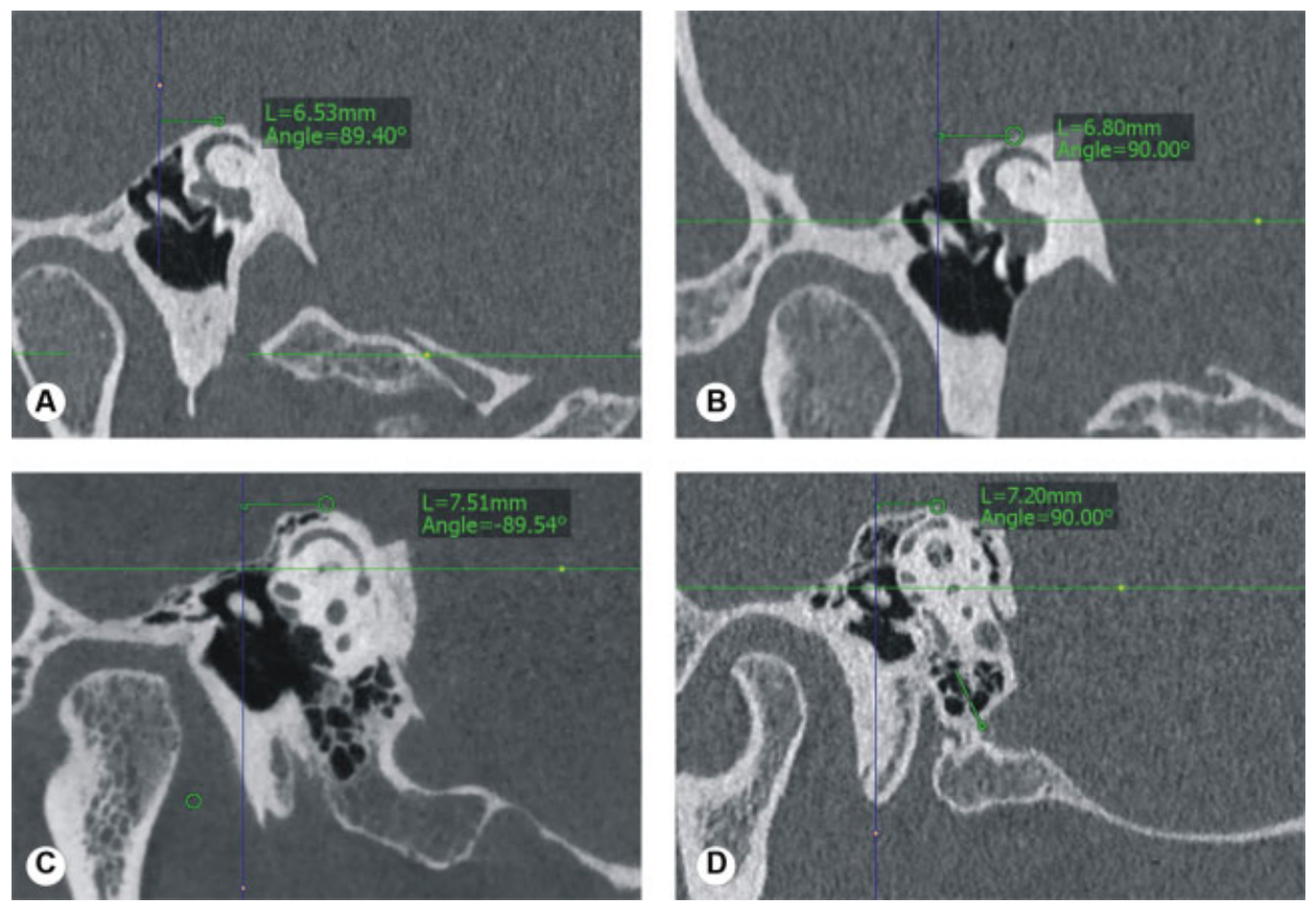

Fig. 7 Measurement of the distance between the SCC and the IMJ in reformatted scans. Abbreviation: L: distance between the SCC and the IMJ midpoints. Note the presence of air cells superior to the SSC can confuse the surgeon searching for the blue line of the SCC in scans C and D. 
Table 1 Middle fossa approach measurements

\begin{tabular}{|l|l|l|l|l|l|}
\hline TEMPORAL BONES DISSECTED & DIM] $(\mathrm{cm})$ & ATTW $\left(\mathrm{mm}^{2}\right)$ & CT scan No & DAS $(\mathrm{mm})$ & DDR $(\mathrm{mm})$ \\
\hline 1. & 1.75 & 35 & I. & 7.68 & 7.03 \\
\hline 2. & 1.71 & 30 & II. & 7.77 & 7.02 \\
\hline 3. & 1.8 & 84 & III. & 6.73 & 5.93 \\
\hline 4. & 1.7 & 32 & IV. & 6.37 & 5.17 \\
\hline 5. & 1.7 & 24 & V. & 6.94 & 5.52 \\
\hline 6. & 1.7 & 90 & VI. & 7.48 & 6.78 \\
\hline 7. & 1.9 & 72 & VII. & 8.85 & 7.39 \\
\hline 8. & 1.7 & 60 & VIII. & 7.20 & 6.29 \\
\hline 9. & 1.8 & 60 & IX. & 8.49 & 7.19 \\
\hline 10. & 1.9 & 100 & X. & 6.96 & 6.07 \\
\hline 11. & 1.8 & 28 & XI. & 8.72 & 7.53 \\
\hline 12. & 1.8 & 24 & XII. & 7.87 & 7.32 \\
\hline 13. & 1.8 & 120 & XIII. & 7.67 & 7.20 \\
\hline 14. & 1.7 & 70 & XIV. & 8.25 & 7.6 \\
\hline 15. & 1.6 & 25 & XV. & 8.03 & 7.51 \\
\hline 16. & 1.9 & 88 & XVI. & 8.49 & 7.4 \\
\hline 17. & 1.8 & 50 & XVII. & 7.54 & 6.8 \\
\hline 18. & 1.8 & 36 & XVIII. & 7.78 & 6.53 \\
\hline 19. & 1.7 & 80 & XIX. & 7.9 & 7.53 \\
\hline 20. & 1.85 & 72 & XX. & 8.06 & 7.49 \\
\hline RANGE & $1.6-1.9$ & $24-120$ & -1 & $6.37-8.85$ & $5.17-7.6$ \\
\hline MEAN & 1.7705 & 59 & - & 7.739 & 6.865 \\
\hline STANDARD DEVIATION & 0.081336 & 29.06798 & - & 0.663411 & 0.722273 \\
\hline
\end{tabular}

Abbreviations: AIM], angle between incudomalleal joint and petrous line; ATTW, area of tegmen tympani window; D, distance between the blue line of the superior semicircular canal and the incudomalleal joint (IMJ) line; DAS, distance from midpoint of IMJ to superior most part of superior semicircular canal (SSC) in axial CT scan; DDR, distance from midpoint of IMJ to superior most part of SSC in 3 D reconstructed paracoronal images; DIMJ, distance of the IMJ from the outer cortical level at the root of zygoma.

bone to be removed before the blue-line is exposed. Sixty degrees is the "safe angle" to search for the IAC and/or the facial nerve immediately below the meatal plane in a narrow surgical field, thereby circumventing the need to take numerous measurements with a navigation system.

Criticism has been generated on the technique of opening the tegmen, because the defect creates potential routes for cerebrospinal fluid (CSF) leak or meningeal prolapse, which may lead to conductive hearing loss. ${ }^{13,14}$ However, over the last 2 decades of uninterrupted clinical practice, the senior author has not had a single case of CSF leak, meningitis, or conductive hearing loss thanks to standardization of the surgical technique and systematically reconstructing the small tegmen defect in all cases. Other surgeons with a vast experience on MCF surgery proposed to rely on the anatomy of the middle meningeal artery, the greater superficial petrosal nerve and the arcuate eminence. ${ }^{15}$ In our experience, the dissection close to the foramen spinosum leads to increased bleeding from surrounding dural vessels, and the greater superficial petrosal nerve may run in a bony channel, not easily visible toward the surgeon. It often also requires an enlarged craniotomy and may be better suited for vestibular schwannomas with a limited extension into the cerebellopontine angle of less than $5 \mathrm{~mm} .{ }^{16}$

\section{Conclusions}

The IMJ is a safe and consistent anatomical marker in the surgical approach to the MCF to identify the SSC, a critical landmark for the middle fossa approach. The IMJ can be located by drilling into the tegmen 1.5 to $2 \mathrm{~cm}(1.6-1.9 \mathrm{~cm}$ in our study) medial to the ROZ posteriorly. The SCC can be traced along the virtual line from the ROZ crossing the IMJ line and extending posteriorly. The superior most aspect of the SCC lies within 0.5 to $1 \mathrm{~cm}$ from the IMJ. Knowledge of the close relationship between the direction of the IMJ and SSC can be used in the SSCD surgery to correctly identify the area of dehiscence. In the MCFA, this relationship and the blue lining of the SCC can direct the surgeon to the IAC. We strongly recommend to always analyze the shape of the tegmen and the degree of pneumatization within the arcuate eminence above the SSC in preoperative CT scans, orienting the surgeon in a rather narrow field with limited retraction of the dura and brain. 


\section{Conflict of Interests}

The authors have no conflict of interests to declare.

\section{References}

1 Yetiser S. Total facial nerve decompression for severe traumatic facial nerve paralysis: a review of 10 cases. Int J of Otolaryngol. Volume 2012, 2012:607359. doi:10.1155/2012/607359. Epub 2011 Nov 20

2 Domenech Juan I, Cruz Toro P, Callejo Castillo Á, Moya R, Merán Gil JL, Bartel R. Middle fossa approach: Applications in temporal bone lesions. Abordaje por fosa media, aplicaciones en lesiones del hueso temporal. Acta Otorrinolaringol Esp. 2016;67(04):233238. doi:10.1016/j.otorri.2015.06.001

3 Peng KA, Lekovic GP, Wilkinson EP. Pearls for the middle fossa approach in acoustic neuroma surgery. Curr Opin Otolaryngol Head Neck Surg 2018;26(05):276-279

4 Mikulec AA, Poe DS, McKenna MJ. Operative management of superior semicircular canal dehiscence. Laryngoscope 2005;115 (03):501-507

5 Fisch U. Transtemporal surgery of the internal auditory canal. Transtemporal Surgery of the Internal Auditory Canal. 17: Karger Publishers; 1970:203-240

6 Fisch U, Mattox D. Microsurgery of the skull base. Thieme; 1988

7 Tsunoda A, Kimura Y, Sumi T, Komatsuzaki A, Sato T. The arcuate eminence is not a protrusion of the superior semi-circular canal but a trace of sulcus on the temporal lobe. J Laryngol Otol 2000; 114(05):339-344

8 Seo Y, Ito T, Sasaki T, Nakagawara J, Nakamura H. Assessment of the anatomical relationship between the arcuate eminence and superior semicircular canal by computed tomography. Neurol Med Chir (Tokyo) 2007;47(08):335-339, discussion 339340

9 El Hadi T, Sorrentino T, Calmels M-N, Fraysse B, Deguine O, Marx M. Spontaneous tegmen defect and semicircular canal dehiscence: same etiopathogenic entity? Otol Neurotol 2012;33(04): 591-595

10 Patel NS, Hunter JB, O'Connell BP, Wanna GB, Carlson ML. O'ConnellBP, WannaGB, CarlsonML. An easy and reliable method to locate the dehiscence during middle fossa superior canal dehiscence surgery: it is a (C) inch. Otol Neurotol 2016;37(08):1092-1095

11 Maina R, Ducati A, Lanzino G. The middle cranial fossa: morphometric study and surgical considerations. Skull Base 2007;17(06): 395-403

12 Beckett JS, Chung LK, Lagman C, et al. A Method of locating the dehiscence during middle fossa approach for superior semicircular canal dehiscence surgery. J Neurol Surg B Skull Base 2017;78 (04):353-358

13 Sennaroglu L, Slattery WH III. Petrous anatomy for middle fossa approach. Laryngoscope 2003;113(02):332-342

14 Cokkeser Y, Aristegui M, Naguib MB, Saleh E, Taibah AK, Sanna M. Identification of internal acoustic canal in the middle cranial fossa approach: a safe technique. Otolaryngol Head Neck Surg 2001; 124(01):94-98

15 Garcia-Ibanez E, Garcia-Ibanez JL. Middle fossa vestibular neurectomy: a report of 373 cases. Otolaryngol Head Neck Surg 1980; 88(04):486-490

16 House WF, Shelton C. Middle fossa approach for acoustic tumor removal. 1992. Neurosurg Clin N Am 2008;19(02):279-288, vi vi 